\title{
Quantum-mechanical versus semiclassical calculations of dc-field-induced tunneling rates for helium for field strengths in the range $0.067-1.0$ a.u.
}

\author{
Spyros I. Themelis, ${ }^{1}$ Theodoros Mercouris, ${ }^{1}$ and Cleanthes A. Nicolaides ${ }^{1,2}$ \\ ${ }^{1}$ Theoretical and Physical Chemistry Institute, National Hellenic Research Foundation, 48 Vassileos Constantinou Avenue, \\ 11635 Athens, Greece \\ ${ }^{2}$ Physics Department, National Technical University, Athens, Greece
}

(Received 9 August 1999; published 20 December 1999)

\begin{abstract}
The dc-field-induced tunneling rates for He were computed semiclassicall,y and quantum mechanically, the latter case being the result of the application of our previously published nonperturbative theory which accounts for electronic structure, electron correlation, and field-induced effects. As the field strength increases beyond 0.15 a.u., the deviation between the two calculations is enhanced, a phenomenon which is intrinsic to the WKB approximation and is observed explicitly, via the known expressions, in the hydrogen atom.
\end{abstract}

PACS number(s): 03.65.Sq, 32.60.+i, 31.15.Ar

Consider an atomic state, ground or excited, interacting with a static electric or magnetic field whose strength is not negligible relative to the binding energy of the outer active electrons. The energy of the state is shifted and its width is altered as a result of this interaction, as a function of field strength. The related problem then is how to compute from first principles these changes to all orders in perturbation theory, as regards both the interelectronic interactions and the field-atom coupling.

Because of the formal and computational difficulty of dealing simultaneously with both these conditions, theoretical constructions and calculations over many decades since the invention of quantum mechanics were restricted to oneelectron systems and to semiclassical models. In a series of papers from this institute it has been shown how this problem can be dealt with nonperturbatively for polyelectronic states, for electric [see, e.g., Refs. [1-3]] as well as for magnetic [4] fields. The applications have involved ground states, singly excited discrete states, and doubly excited resonance states of neutral atoms and of negative ions.

Once the possibility of $a b$ initio many-electron calculation exists, it is useful to compare with models that are easily used in order to understand the range and conditions of their applicability. In two such examples [3] we have compared our results on $\mathrm{He}, \mathrm{H}, \mathrm{Li}^{-}$, and $\mathrm{Li}$ ground and low-lying excited states with the semiclassical models introduced over the years for static [5-7] and cycle-averaged low-frequency electric fields [8]. The latter, known as the AmmosovDelone-Krainov (ADK) formula, is an extension of the formulas given in Refs. [5-7], and has been used in recent years for heuristic purposes in the interpretation of strong field dynamics (see, e.g., Refs. [9], [10]).

In this Brief Report we present results from $a b$ initio quantum-mechanical calculations on $\mathrm{H}$ and $\mathrm{He}$ for the dcfield-induced tunneling rates (FITR) and from the use of semiclassical formulas, for a broad range of field strengths, in continuation of the work published in Ref. [3]. After the completion of our calculations, a paper on this topic was published recently [11]. The calculations of Ref. [11] were done by applying the complex coordinate rotation (CCR) method $[12,13]$. The results of Ref. [11] are in agreement with the present ones (Table I), which were obtained via the state-specific theory for the solution of the complex eigenvalue Schrödinger equation (see e.g., Refs. [1-4], and references therein). Furthermore, we have also covered a region with small values of the field strength, not calculated in Ref. [11]. Calculations for this region were first reported in Ref. [3]. However, due to the computer limitations at the time, the function spaces and the parameter optimization were not sufficiently flexible, and led the calculations to falsely converged results which are replaced by the present ones.

The theory and methods employed for the present nonperturbative calculations have been reported in the past (see, e.g., Refs. [1-4]), and so here only the characteristics of the calculation on He will be given. The bare ${ }^{1} S \mathrm{He}$ state was represented by a state-specific correlated wave function, obtained as described in Ref. [14] and references therein. A 20 -term expansion with numerical and analytic orbitals up to $\ell=3$ was optimized variationally. The function space, representing localized states which are allowed to mix via the

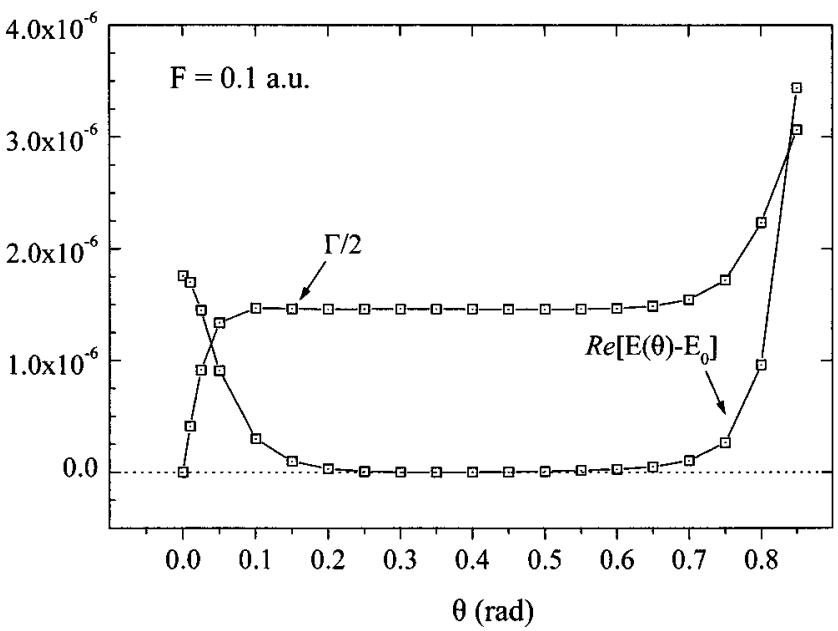

FIG. 1. Plot of the width and of the difference between the real part of the complex eigenvalue $(E(\theta))$ and its final solution $\left(E_{0}\right)$, of $\mathrm{He} 1 s^{2}{ }^{1} S$, for $F=0.1$ a.u., vs the rotation angle $\theta$ present in the complex Slater-type orbitals. 
TABLE I. Field-induced tunneling width for $\mathrm{He} 1 s^{2}{ }^{1} S$ in a.u. The difference between the two quantummechanical calculations is insignificant. On the other hand, the difference between these and the semiclassical calculations increases with increasing field strength.

\begin{tabular}{|c|c|c|c|}
\hline \multirow[b]{3}{*}{ F (a.u.) } & \multicolumn{3}{|c|}{$\Gamma($ a.u. $)$} \\
\hline & \multicolumn{2}{|c|}{ Quantum mechanical } & \multirow{2}{*}{$\begin{array}{c}\text { Semiclassical } \\
\text { formula of Ref. [7] }\end{array}$} \\
\hline & This work & Ref. [11] & \\
\hline 0.067 & $1.6 \times 10^{-9}$ & & $9.51 \times 10^{-10}$ \\
\hline 0.068 & $2.0 \times 10^{-9}$ & & $1.35 \times 10^{-9}$ \\
\hline 0.069 & $2.7 \times 10^{-9}$ & & $1.89 \times 10^{-9}$ \\
\hline 0.070 & $3.6 \times 10^{-9}$ & & $2.62 \times 10^{-9}$ \\
\hline 0.071 & $4.6 \times 10^{-9}$ & & $3.61 \times 10^{-9}$ \\
\hline 0.072 & $6.2 \times 10^{-9}$ & & $4.92 \times 10^{-9}$ \\
\hline 0.073 & $8.2 \times 10^{-9}$ & & $6.65 \times 10^{-9}$ \\
\hline 0.074 & $1.08 \times 10^{-8}$ & & $8.92 \times 10^{-9}$ \\
\hline 0.075 & $1.42 \times 10^{-8}$ & & $1.19 \times 10^{-8}$ \\
\hline 0.076 & $1.86 \times 10^{-8}$ & & $1.57 \times 10^{-8}$ \\
\hline 0.077 & $2.35 \times 10^{-8}$ & & $2.05 \times 10^{-8}$ \\
\hline 0.078 & $3.18 \times 10^{-8}$ & & $2.67 \times 10^{-8}$ \\
\hline 0.079 & $4.25 \times 10^{-8}$ & & $3.85 \times 10^{-8}$ \\
\hline 0.080 & $5.57 \times 10^{-8}$ & $4.63 \times 10^{-8}$ & $4.44 \times 10^{-8}$ \\
\hline 0.082 & $9.28 \times 10^{-8}$ & & $7.18 \times 10^{-8}$ \\
\hline 0.084 & $1.49 \times 10^{-7}$ & & $1.14 \times 10^{-7}$ \\
\hline 0.086 & $2.32 \times 10^{-7}$ & & $1.76 \times 10^{-7}$ \\
\hline 0.088 & $3.52 \times 10^{-7}$ & & $2.67 \times 10^{-7}$ \\
\hline 0.090 & $5.23 \times 10^{-7}$ & $5.09 \times 10^{-7}$ & $3.97 \times 10^{-7}$ \\
\hline 0.095 & $1.30 \times 10^{-6}$ & & $9.98 \times 10^{-7}$ \\
\hline 0.10 & $2.92 \times 10^{-6}$ & $2.88 \times 10^{-6}$ & $2.28 \times 10^{-6}$ \\
\hline 0.11 & $1.17 \times 10^{-5}$ & $1.15 \times 10^{-5}$ & $9.51 \times 10^{-6}$ \\
\hline 0.12 & $3.66 \times 10^{-5}$ & $3.62 \times 10^{-5}$ & $3.11 \times 10^{-5}$ \\
\hline 0.13 & $9.50 \times 10^{-5}$ & $9.43 \times 10^{-5}$ & $8.44 \times 10^{-5}$ \\
\hline 0.14 & $2.13 \times 10^{-4}$ & $2.12 \times 10^{-4}$ & $1.98 \times 10^{-4}$ \\
\hline 0.15 & $4.25 \times 10^{-4}$ & $4.23 \times 10^{-4}$ & $4.15 \times 10^{-4}$ \\
\hline 0.16 & $7.70 \times 10^{-4}$ & $7.68 \times 10^{-4}$ & $7.89 \times 10^{-4}$ \\
\hline 0.18 & $2.03 \times 10^{-3}$ & $2.03 \times 10^{-3}$ & $2.30 \times 10^{-3}$ \\
\hline 0.20 & $4.30 \times 10^{-3}$ & $4.31 \times 10^{-3}$ & $5.36 \times 10^{-3}$ \\
\hline 0.25 & $1.56 \times 10^{-2}$ & $1.57 \times 10^{-2}$ & $2.43 \times 10^{-2}$ \\
\hline 0.30 & $3.52 \times 10^{-2}$ & $3.56 \times 10^{-2}$ & $6.55 \times 10^{-2}$ \\
\hline 0.35 & $6.25 \times 10^{-2}$ & $6.33 \times 10^{-2}$ & $1.31 \times 10^{-1}$ \\
\hline 0.40 & $9.64 \times 10^{-2}$ & $9.77 \times 10^{-2}$ & $2.20 \times 10^{-1}$ \\
\hline 0.45 & $1.37 \times 10^{-1}$ & $1.38 \times 10^{-1}$ & $3.25 \times 10^{-1}$ \\
\hline 0.50 & $1.88 \times 10^{-1}$ & $1.83 \times 10^{-1}$ & $4.43 \times 10^{-1}$ \\
\hline 0.60 & $2.70 \times 10^{-1}$ & $2.87 \times 10^{-1}$ & $6.95 \times 10^{-1}$ \\
\hline 0.70 & $3.92 \times 10^{-1}$ & $4.06 \times 10^{-1}$ & $9.48 \times 10^{-1}$ \\
\hline 0.80 & $5.24 \times 10^{-1}$ & $5.36 \times 10^{-1}$ & $11.86 \times 10^{-1}$ \\
\hline 0.90 & $6.75 \times 10^{-1}$ & $6.73 \times 10^{-1}$ & $14.03 \times 10^{-1}$ \\
\hline 1.00 & $8.50 \times 10^{-1}$ & $8.18 \times 10^{-1}$ & $15.95 \times 10^{-1}$ \\
\hline
\end{tabular}

interactions of the total Hamiltonian (the Hamiltonian coordinates in this theory remain real), included Rydberg configurations $1 \operatorname{sn} \ell{ }^{1} L$, where $n=2-6$ and $\ell=0,1, \ldots, n-1$, and doubly excited configurations $v_{\ell} v_{\ell}^{\prime},{ }^{1} L$, where $v_{\ell}$ are analytic virtual orbitals with $\ell, \ell^{\prime}=0,1,2,3$ and ${ }^{1} L$ $={ }^{1} S,{ }^{1} P,{ }^{1} D,{ }^{1} F$, and ${ }^{1} G$ of even and odd parity. As regards the function space representing the asymptotic openchannel part, this was represented by $1 s \varepsilon \ell$ configurations, where each $\varepsilon \ell$ is expanded in terms of complex Slatertype orbitals (STO's) $\varphi_{\kappa}=r^{\kappa} e^{-\alpha r e^{-i \theta}}$. For these states, $\ell$ $\leqslant 5$. "Continuum', states with $\ell \leqslant 4$ were represented by ten complex STO's, while for $\ell=5$ we used eight complex STO's. The convergence of our results for the FITR with respect to the nonlinear parameters of the STO's was tested for each value of the field strength. The optimal value of the parameter $\alpha$ was $\alpha=1.5$. As an example of the type of con- 


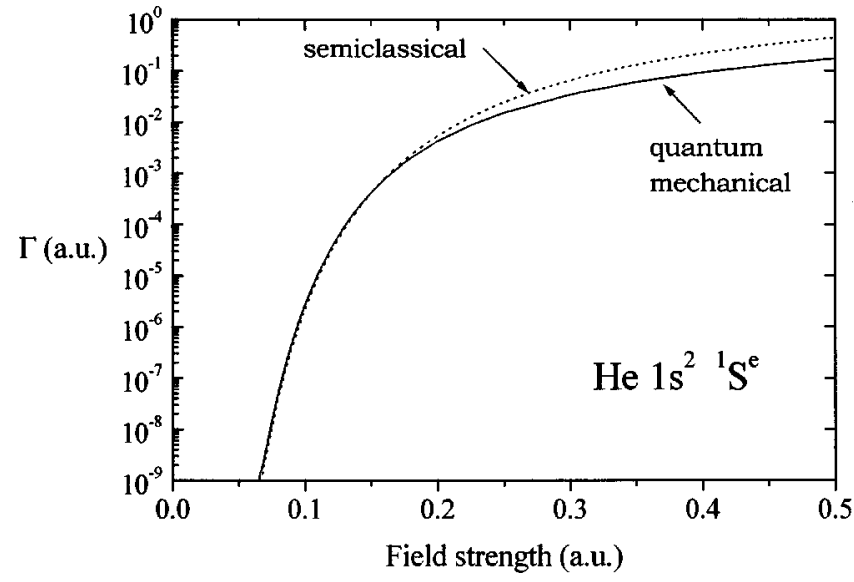

FIG. 2. Graphical comparison of the results given in Table I.

vergence obtained in the state-specific calculations, in Fig. 1 we plot the real and imaginary part of the complex eigenvalue versus the angle $\theta$, for $F=0.1$ a.u.

The results of our calculation are given in Table I and Fig. 2 , where we also include the results from the WKB formula of Ref. [7], using the constant given in Ref. [8], and the recently published CCR results of Ref. [11]. The agreement of our results with those of Ref. [11] is very good. The results from the semiclassical formula start to deviate as the field strength increases beyond 0.15 a.u. This discrepancy gave us the opportunity to comment as follows:

For the hydrogen atom, the FITR was obtained analytically to a good approximation quantum mechanically, using perturbation theory (PT) $[15,16]$ as well as semiclassically, using the WKB approximation [6]. According to the former, the width for the $1 s$ state has the form

$$
\Gamma_{H}(F)=\frac{4}{F} e^{-2 / 3 F}\left[1-\frac{107}{12} F+\frac{7363}{288} F^{2}-\cdots\right] .
$$

The WKB approximation gives only the first term of this expansion. It is clear that as the field strength increases, the deviation between the prediction of Eq. (1) and that of the WKB approximation increases. At the same time, more and more polynomial terms become necessary as the field

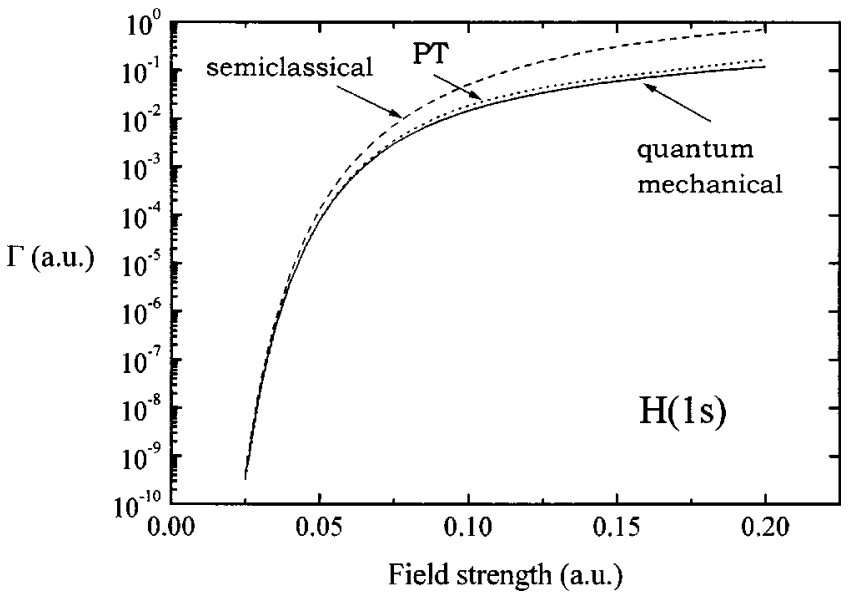

FIG. 3. dc-field-induced tunneling widths for $H$, in a.u. The PT results are obtained from Eq. (1), while the semiclassical ones by using only the first term. The accurate quantum-mechanical results were calculated by the present nonperturbative theory and by the large order perturbation theory of Ref. [17].

strength increases, albeit to unphysically high values. These facts are depicted in Fig. 3, which compares three types of calculation. The exact quantum-mechanical results are taken from the large order perturbation theory calculations of Silverman and Nicolaides [17] and from the present calculations.

An asymptotic expansion similar to Eq. (1) is valid for a model potential which was used to describe the FITR of bound negative ions [18]. Therefore we can conclude, as Delone and Krainov also did [19], that the semiclassical formula has corrections of the form $\left(1+c_{1} F+c_{2} F^{2}+c_{3} F^{3}\right.$ $+\cdots)$. The coefficients of this series are in general large numbers, asymptotic in the parameter $2\left(2 E_{b}\right)^{3 / 2} / 3$, where $E_{b}$ is the binding energy. This correction is in general not known for many-electron systems. However, it can be expected that the deviation between quantum mechanics and semiclassical mechanics will appear to be enhanced for states of small binding energy, since the coefficients $c_{i}$ depend on inverse powers of $E_{b}$ [3].

One of us (S. I. T.) expresses his gratitude to the A. Onassis Foundation, Athens, Greece, for financial support.
[1] C. A. Nicolaides and Th. Mercouris, Chem. Phys. Lett. 159, 45 (1989).

[2] Th. Mercouris and C. A. Nicolaides, J. Phys. B 24, L557 (1991).

[3] S. I. Themelis and C. A. Nicolaides, Phys. Rev. A 49, 3089 (1994); 59, 2500 (1999).

[4] M. Bylicki, S. I. Themelis, and C. A. Nicolaides, J. Phys. B 27, 2741 (1994).

[5] Yu. N. Demkov and G. F. Drukarev, Zh. Eksp. Teor. Fiz. 47, 918 (1964) [Sov. Phys. JETP 20, 614 (1965)].

[6] B. M. Smirnov and M. I. Chibisov, Zh. Eksp. Theor. Fiz. 49, 841 (1965) [Sov. Phys. JETP 22, 585 (1966)].

[7] A. M. Perelomov, V. S. Popov, and M. V. Terent'ev, Zh. Eksp.
Teor. Fiz. 50, 1393 (1966) [Sov. Phys. JETP 23, 924 (1966)].

[8] M. V. Ammosov, N. B. Delone, and V. P. Krainov, Zh. Eksp. Teor. Fiz. 91, 2008 (1986) [Sov. Phys. JETP 64, 1191 (1986)].

[9] P. B. Corkum, N. H. Burnett, and F. Brunel, Phys. Rev. Lett. 62, 1259 (1989).

[10] G. Gibson, T. S. Luk, and C. K. Rhodes, Phys. Rev. A 41, 5049 (1990).

[11] A. Scrinzi, M. Geissler, and T. Brabec, Phys. Rev. Lett. 83, 706 (1999).

[12] G. D. Doolen, J. Phys. B 8, 525 (1975).

[13] W. P. Reinhardt, Int. J. Quantum Chem., Symp. 10, 359 (1976).

[14] C. A. Nicolaides, Int. J. Quantum Chem. 60, 119 (1996). 
[15] R. J. Damburg and V. V. Kolosov, J. Phys. B 11, 1921 (1978).

[16] H. J. Silverstone, B. G. Adams, J. Cizek, and P. Otto, Phys. Rev. Lett. 43, 1498 (1979).

[17] J. N. Silverman and C. A. Nicolaides, Chem. Phys. Lett. 153,
61 (1988); 184, 321 (1991).

[18] N. A. Guschina and V. K. Nikulin, Chem. Phys. 10, 23 (1975).

[19] N. B. Delone and V. P. Krainov, Usp. Fiz. Nauk 168, 531 (1998) [Sov. Phys. Usp. 41, 469 (1998)]. 